\title{
EL TRASTORNO DE ESPECTRO AUTISTA. NECESIDADES EDUCATIVAS A LA LUZ DEL INTERÉS SUPERIOR DEL NIÑO A PROPÓSITO DE LA SENTENCIA DE LA CORTE DE APELACIONES DE ANTOFAGASTA, DE 6 DE DICIEMBRE DE 2018
}

\author{
AUTISM SPECTRUM DISORDER. \\ EDUCATIONAL NEEDS IN LIGHT OF THE BEST INTEREST OF \\ THE CHILD IN RELATION TO THE RULING OF THE COURT OF \\ APPEALS OF ANTOFAGASTA, PASSED ON DECEMBER 6, 2018
}

CONSTANZA ASTUDILLO MEZA*

ALEXIS MONDACA MIRANDA**

\section{RESUMEN}

En el presente trabajo se analiza la sentencia de la Corte de Apelaciones de Antofagasta, del 6 de diciembre de 2018, que resuelve un recurso de protección interpuesto por la madre de un niño con trastorno del espectro autista, en contra de su establecimiento educacional, que decidió no renovar la matrícula de su hijo por causa de su condición.Lo anterior, sobre la base de las exigencias planteadas por el principio del interés superior del niño,

\footnotetext{
* Magister en Derecho por la Universidad Católica del Norte. Profesora de Derecho Civil de la Facultad de Derecho de la Universidad Santo Tomás, Antofagasta, Chile. Correo electrónico: castudillom@ santotomas.cl. ORCID: https://orcid.org/0000-0001-9796-3492.

** Doctor en Derecho por la Pontificia Universidad Católica de Valparaíso. Máster en Derecho, Empresa y Justicia por la Universidad de Valencia. Profesor de Derecho Civil de la Facultad de Ciencias Jurídicas y Sociales de la Universidad de Talca, Chile. Correo electrónico: alexis.mondaca@utalca.cl. ORCID: https://orcid.org/0000-0002-6559-4124.

Trabajo recibido para su evaluación el 30 de julio de 2019, y aceptado para su publicación el 18 de junio de 2020.
} 
considerando las necesidades educativas particulares derivadas del trastorno del espectro autista. Se resalta el deber de no discriminar a los niños, niñas $\mathrm{y}$ adolescentes que necesitan una educación apropiada a sus condiciones especiales.

Palabras clave: interés superior del niño, recurso de protección, arbitrariedad, ilegalidad, trastorno del espectro autista, discriminación.

\section{ABSTRACT}

The present paper analyzes the ruling passed on December 6th of 2018 by the Court of Appeals of Antofagasta, wich resolves a protection action brought by the mother of a child with autism spectrum disorder, against his educational establishment, who decided not to renew the registration of his son because of his condition. The above, on the basis of the requirements imposed by the principle of the best interest of the child, considering the particular educational needs derived from the autism spectrum disorder. The duty of non-discrimination of children and adolescents in need of adequate education in accordance with their special conditions is highlighted.

Keywords: best interests of the child, protection action, arbitrariness, illegality, autism spectrum disorder, discrimination.

\section{DOCTRINA}

No corresponde cancelar la matrícula de un alumno que presenta un trastorno del espectro autista. Dicho actuar es arbitrario, porque si un estudiante es admitido por un establecimiento educacional, a sabiendas de su particular condición, no es aceptable que, con posterioridad, basándose en la misma condición, se tome una medida tan drástica como la mencionada. La ilegalidad se funda en la violación de los artículos 34 y 36 de la Ley $\mathrm{N}^{\mathrm{o}} 20.422$, preceptos que garantizan la educación de los alumnos con necesidades educativas especiales.

Corte de Apelaciones de Antofagasta, 6 de diciembre de 2018, Rol No 3.149-2018. 


\section{COMENTARIOS}

\section{Interés superior del niño y necesidades educativas especiales}

El principio del interés superior del niño se regula en el artículo 3.1 de la Convención sobre los Derechos del Niño en el siguiente sentido: "En todas las medidas concernientes a los niños que tomen las instituciones públicas o privadas de bienestar social, los tribunales, las autoridades administrativas o los órganos legislativos, una consideración primordial a que se atenderá será el interés superior del niño". Siguiendo a Cillero, esta supremacía del interés superior del niño implica que debe primar sobre consideraciones culturales y cálculos de beneficio colectivo. ${ }^{1}$

La doctrina nacional entiende que el interés superior del niño es un concepto jurídico indeterminado. Es decir, según LATHROP, dicha noción está en desarrollo, lo que permite calificarle como concepto indeterminado, relativo o abstracto, que necesita ser concretado en cada caso. ${ }^{2}$ Así, como lo indica Aguilar, el nombrado instrumento internacional consagra el principio en comento, "pero no explica o define qué se debe entender por interés superior del niño. En este sentido, ha sido rol de la doctrina y de la jurisprudencia conceptualizar y establecer los límites y alcances de éste". ${ }^{3}$ En la misma línea, indican RavetLlat y PinOchet, que una correcta aplicación del principio del interés superior debe considerar las particularidades de cada caso, entre éstas, las características de los sujetos intervinientes, junto con la correspondiente evolución social y jurídica. ${ }^{4}$

Súmese a lo anterior, la labor de la jurisprudencia; luego, han sido principalmente los tribunales de justicia los que han recogido la noción de la doctrina relativa a que el interés superior es un concepto indeterminado "cuyo alcance se aprecia cuando es aplicado al caso concreto", 5 y en este

\footnotetext{
${ }^{1}$ Cillero, Miguel, "El interés superior del niño en el marco de la Convención Internacional sobre los Derechos del Niño", Justicia y Derechos del Niño, 1999, No 1, p. 48.

${ }^{2}$ Lathrop, Fabiola, Cuidado personal de los hijos. Análisis de la Ley de matrimonio civil y Tribunales de Familia, Punto Lex, Santiago, 2005, p. 33.

${ }^{3}$ Aguilar, Gonzalo, "El principio del interés superior del niño y la Corte Interamericana de Derechos Humanos", Estudios Constitucionales, 2008, ํ 1, Vol. 6, p. 7.

${ }^{4}$ Ravetllat, Isaac; Pinochet, Ruperto, "El interés superior del niño en el marco de la Convención Internacional sobre los Derechos del Niño y su configuración en el Derecho civil chileno", Revista Chilena de Derecho, 2015, Vol. 42, No 3, p. 916.

${ }^{5}$ Corte suprema, 18 de mayo de 2016, considerando $11^{\circ}$, Rol 35.522-2015.
} 
trabajo, el caso concreto está dado por las necesidades educativas de los niños, niñas y adolescentes (en adelante NNA), que presentan un trastorno del espectro autista (en adelante TEA).

El derecho a la educación permite el ejercicio de todos los demás derechos humanos, en palabras del Comité de Derechos Económicos, Sociales y Culturales de la ONU, es el "epítome de la indivisibilidad y la interdependencia de todos los derechos humanos". ${ }^{6}$ Por lo señalado, es fundamental que existan las condiciones para que dicho derecho se aplique de manera plena respecto de los NNA.

Creemos que estas condiciones son propiciadas a través de la evaluación y determinación ${ }^{7}$ del interés superior del niño. Así, en el caso que analizamos, mediante la ponderación de las circunstancias concretas de cada NNA, podremos concluir que mantener el entorno educativo con compañeros y profesores respecto de un NNA que padece de un TEA, contribuye a su mejor interés.

Lo anterior es coherente con la Observación General $N^{\circ} 1$ del Comité de los Derechos del Niño, referida al derecho a la educación, en cuya virtud la educación "es una actividad singular que debe realizarse en cada caso, teniendo en cuenta las circunstancias concretas de cada niño (...). Esas circunstancias se refieren a las características específicas del niño o los niños de que se trate, como la edad, el sexo, el grado de madurez, la experiencia, la pertenencia a un grupo minoritario, la existencia de una discapacidad física, sensorial o intelectual y el contexto social y cultural del niño o los niños". ${ }^{8}$

Por lo tanto, la construcción del interés superior en los NNA con necesidades educativas especiales, en concreto TEA, no puede obviar que el objetivo principal de la educación "es el desarrollo de la personalidad de cada niño, de sus dotes naturales y capacidad, reconociéndose el hecho de que cada niño tiene características, intereses y capacidades únicas y también

\footnotetext{
${ }^{6}$ Comité de Derechos Económicos, Sociales y Culturales ONU, Observación No 11 (1999), Planes de acción para la enseñanza primaria (artículo 14 del Pacto), Naciones Unidas, Nueva York, 2000. Según LePín, el principio del interés superior del niño posibilita la efectividad de otros principios o derechos vinculados a los NNA, por lo que es un principio general de la legislación, LEPIN, Cristian, "Los nuevos principios del Derecho de Familia", Revista Chilena de Derecho Privado, 2014, N²3, p. 39.

${ }^{7}$ Comité de Derechos del Niño UNICEF, Observación General No 14 (2013), sobre el derecho del niño a que su interés superior sea una consideración primordial (artículo 3, párrafo 1), Naciones Unidas, Nueva York, 2013, p. 10.

${ }^{8}$ Comité de Derechos del Niño (UNICEF), Observación General $N^{\circ} 1$ (2001), Propósitos de la educación, Naciones Unidas, Nueva York, 2001, p. 8.
} 
necesidades de aprendizaje propias". ${ }^{9}$ En la misma senda, según SilveirA, el principio del interés superior del niño "ha de tenerse en cuenta en cada una de las decisiones que se tomen respecto a las trayectorias educativas y sobre todo en aquellas donde la complejidad que las atraviesa pareciera oficiar a modo de obstáculo para habilitar, alojar e incluir". ${ }^{10}$ Así, como lo ha planteado BARCIA, el interés superior del niño reconoce como uno de sus principales ámbitos de aplicación la protección de la infancia, "en cuyo caso se centra en niños vulnerables", como los que tienen un TEA. ${ }^{11}$

\section{Del Trastorno del Espectro Autista}

El "TEA", ${ }^{12}$ en términos generales, es un trastorno que afecta el neurodesarrollo y está caracterizado por provocar una serie de dificultades en la comunicación y en la interacción social, ${ }^{13}$ así como por la existencia de un comportamiento estereotipado ${ }^{14}$ y repetitivo. Estas especiales condiciones

\footnotetext{
${ }^{9}$ Comité de Derechos del Niño (UNICEF), cit. (n. 8), p. 7.

${ }^{10}$ SILVEIRA, Israel, "La aplicación del interés superior del niño en el ámbito educativo. Las concepciones de los profesionales de los equipos de orientación escolar", XXIII Jornadas de Investigación. XII Encuentro de Investigadores en Psicología del MERCOSUR, Facultad de Psicología Universidad de Buenos Aires, Buenos Aires, 2016, p. 2, en línea: www.aacademica.org/000-044/455.pdf, consultada: 30 de junio de 2019.

${ }^{11}$ BARCIA, Rodrigo, "La evolución de la custodia unilateral conforme a los principios de interés superior del niño y corresponsabilidad de los padres", Ius et Praxis, 2018, No 2, p. 481.

${ }^{12}$ La etiqueta TEA parece remitir a un conjunto heterogéneo de individualidades, cuyos niveles evolutivos, necesidades educativas, terapéuticas y perspectivas vitales son en extremo diversas. Es decir, todas las personas con TEA son diferentes, no están caracterizadas uniformemente por los rasgos de estos trastornos, sino que por el ambiente en el que habitan, las experiencias que tienen en sus vidas $\mathrm{y}$, sobre todo, por los apoyos que tengan para superar las dificultades de estas experiencias y, en su caso, por los modelos educativos a los que estén sujetos, véase a BAÑA, Manoel, "El rol de la familia en la calidad de vida y la autodeterminación de las personas con trastorno del espectro del autismo", Ciencia Psicológica, 2015, Vol. 9, N² 2, p. 323.

${ }^{13}$ El TEA se caracteriza por deficiencias persistentes en la comunicación y en la interacción social en distintos contextos. Lo señalado se concreta en varias insuficiencias: en la reciprocidad socioemocional; en las conductas comunicativas no verbales utilizadas en la interacción social; y en el desarrollo, mantenimiento y comprensión de las relaciones. Súmese a lo señalado la existencia de patrones restrictivos y repetitivos de comportamiento, intereses y actividades. Todo lo dicho puede apreciarse en las primeras fases de desarrollo del TEA de que se trate, Asociación Americana de PsiQuiatría (Eds.), Guía de consulta de los criterios diagnósticos del DSM 5, American Psychiatric Publishing, Arlington, 2014, en línea: http://www.eafit.edu.co/ninos/reddelaspreguntas/Documents/dsm-v-guiaconsulta-manual-diagnostico-estadistico-trastornos-mentales.pdf, consultada: 27 de julio de 2019.

${ }^{14} \mathrm{El}$ comportamiento repetitivo se refleja normalmente en forma de estereotipias, intereses circunscritos, compulsiones y problemas graves de conducta, revísese: MarTínez, Agustín; PiQueras, José, “Conductas repetitivas en personas con trastorno del espectro autista: relación con los antecedentes familiares y el tratamiento farmacológico", Revista Nacional e Internacional de Educación Inclusiva, 2017, № 1,
} 
de NNA en edad escolar, desafían a los establecimientos educacionales, quienes debiesen orientar sus programas y metodologías a los estudiantes que tengan diagnosticado un TEA, ya que una actitud positiva y proactiva de los colegios constituye una clara manifestación del deber del Estado de Chile de velar por el principio del interés superior del niño.

Lo llamativo de la sentencia que comentaremos consiste en que el acto emanado del recurrido afectó a un niño que tiene una especial condición, y en ésta la estabilidad del entorno es determinante. ${ }^{15}$ Podemos decir que el TEA constituye una "condición" que limita o dificulta el desarrollo integral, la incorporación regular de una persona a la sociedad en forma autónoma y su relacionamiento interpersonal, por lo que requiere de apoyo especializado y multisectorial, y, a nivel familiar, de un ambiente comprometido, responsable y dedicado. ${ }^{16}$ Por lo tanto, frente a las características propias de este trastorno que conducen a mantener las circunstancias en que se desenvuelven los NNA que lo padecen, la decisión de un establecimiento educacional de poner fin a la colegiatura basada exclusivamente en la propia existencia de un TEA, resultaría arbitraria e ilegal, además, atentaría en contra del interés superior del niño, según veremos con más detalle en este comentario.

Antes de continuar, debemos recordar al lector que está en presencia de un texto jurídico, sin embargo, hoy la actividad académica y los problemas que ésta debe analizar y resolver requieren de un enfoque interdisciplinario, por ello, nos permitimos ilustrar brevemente a todo el que lea el presente trabajo con nociones técnicas extraídas desde la psicología y psiquiatría. Lo anterior, siguiendo a GonZÁLEZ, puesto que, las "universidades deben autotransformarse, para responder a las nuevas demandas, y generar un alto valor social desde una óptica interdisciplinaria para investigar". ${ }^{17}$

Vol. 10, p. 200, en línea: https://dialnet.unirioja.es/descarga/articulo/6049218.pdf, consultada: 03 de julio de 2019.

${ }^{15}$ Se ha indicado que: “Cómo preparar el contexto educativo para dar respuestas de calidad a la diversidad y a las necesidades educativas especiales? Que la educación eficaz para promover el aprendizaje y lograr el bienestar del niño o niña con autismo y su familia, debe apoyarse en la organización de un contexto natural estable que les permita la comprensión de los sucesos que tienen lugar. Una estabilidad de condiciones que la persona pueda reconocer, sobre las que pueda actuar y que pueda transformar" (el subrayado es nuestro), Ministerio de EduCACión de Chile (Eds.), Guía de apoyo técnico pedagógico para necesidades educativas especiales en el nivel de educación parvularia, Unidad de Educación Especial, Santiago, 2008, 1era Ed., en línea: http://especial.mineduc.cl/wpcontent/uploads/sites/31/2016/08/GuiaAutismo.pdf, consultada: 03 de julio de 2019.

${ }^{16}$ Corte Suprema, 27 de mayo de 2015, Rol N²9.566-2014, considerando $5^{\circ}$.

${ }^{17}$ GonZÁLEZ, Yanesy, "La interdisciplinariedad en la investigación como principio de la responsabilidad social universitaria", Revista Congreso Universitario, 2017, Vol. 6, № 4, p. 128, en línea: http://www. 
Teniendo en vista lo indicado, es necesario señalar que, como lo han estudiado Artigas y PaUla, en el año 1943 el psiquiatra austriaco Leo Karnner resumía la sintomatología del entonces denominado "autismo" -basado en la observación de 11 niños- del siguiente modo: 1) incapacidad para establecer relaciones en el ámbito social; 2) alteraciones en el lenguaje, sobre todo considerado éste como vehículo de comunicación social, aunque en ocho niños el nivel formal de lenguaje era normal o sólo ligeramente retrasado; 3 ) insistencia obsesiva por parte de los niños en mantener el ambiente en el que habitan sin cambios; 4) aparición, en ocasiones, de habilidades especiales, por ejemplo, en el campo de las matemáticas o presentando una memoria particularmente desarrollada; 5) buen potencial cognitivo, pero limitado a sus centros de intereses; 6) aspecto físico normal y "fisonomía inteligente"; y 7) aparición de los primeros síntomas del trastorno desde el nacimiento. ${ }^{18}$

En definitiva, el concepto clásico de autismo "ha variado significativamente desde sus descripciones originales y, en la actualidad, se habla de un continuo, por lo que se prefiere usar el término trastornos del espectro autista (TEA), dada la variable afectación cognitiva y del lenguaje". ${ }^{19}$ Por ello, hoy se habla de "espectro", en el que puede apreciarse que algunas personas tienen uno o más características que recogen los Manuales Diagnósticos elaborados por la Asociación Americana de Psiquiatría, llamados DSM (Diagnostics and Statistics Manual of Mental Disorders). ${ }^{20}$

congresouniversidad.cu/revista/index.php/congresouniversidad/index, consultada: 4 de julio 2019.

${ }^{18}$ Artigas, Joseph; Paula, Isabel, "El autismo 70 años después de Leo Kanner y Hans Asperger", Revista Especializada Neuropsiquiatría, 2011, No 115, Vol. 32, p. 572. Continuando con su estudio, los aludidos autores agregan que, apenas un año después el psiquiatra y pediatra Hans Asperger observó que cuatro niños mostraban un patrón de conducta caracterizado por: "falta de empatía, ingenuidad, poca habilidad para hacer amigos, lenguaje pedante o repetitivo, pobre comunicación no verbal, interés desmesurado por ciertos temas y torpeza motora y mala coordinación. Asperger solía utilizar la denominación de "pequeños profesores" (kleine Professoren) para referirse a ellos, destacando su capacidad para hablar de sus temas favoritos de modo sorprendentemente detallístico y preciso", Ibid., p. 574.

${ }^{19}$ Bonilla, María Fernanda; Chaskel, Roberto, "Trastorno del Espectro Autista", Curso Continuo de Actualización de Pediatría: Sociedad Colombiana de Pediatría, 2016, Vol. 15, No 1, p. 5, en línea: https://scp.com.co/wp-content/uploads/2016/04/2.-Trastorno-espectro.pdf, consultada: 03 de julio de 2019.

${ }^{20}$ Este manual, en conjunto con la Clasificación Internacional de Enfermedades (C-10), son los instrumentos más empleados en psiquiatría para el diagnóstico de enfermedades mentales. El primer DSM fue elaborado en el año 1952 y la última versión es el suplemento del DSM V del año: Asociación Americana de Psiquiatría (Eds.), Diagnostics and Statistics Manual of Mental Disorders, American Psychiatric Publishing, Arlington, 2016, en línea: https://dsm.psychiatryonline.org/pb-assets/dsm/ 


\section{Los hechos}

La madre del niño de iniciales S.E.N.A presentó una acción de protección por el actuar del Colegio Chuquicamata, ubicado en la ciudad de Calama, institución que envió una carta a la recurrente el día 26 de octubre del año 2018, comunicándole en dicha misiva la decisión de no renovar la matrícula de su hijo. Lo último, porque estimó el establecimiento educacional nombrado "que el interés superior de S.E.N.A, podía concretarse y realizarse de mejor manera, en una institución distinta, que contara con mayor especialización e infraestructura adecuada para los reales requerimientos del menor". 21

Ante dicho escenario, manifestó la recurrente que su hijo estudiaba desde el año 2016 en el Colegio Chuquicamata y que presentaba un desempeño satisfactorio respaldado en los informes de aprendizaje emitidos por el propio colegio. Además, se indicó en la acción de protección que el recurrido tomó conocimiento que el niño padecía de un TEA desde el momento de su ingreso al Colegio Chuquicamata, por lo que era arbitraria la medida adoptada por el mencionado colegio. Añadió la recurrente que el recurrido actuó ilegalmente, puesto que se limitó a decretar la expulsión sin aplicar ninguna medida que buscase la continuidad del niño afectado en el Colegio Chuquicamata. En suma, en virtud de todo lo mencionado, la cancelación de la matrícula sería arbitraria e ilegal y constituiría una violación de entidad al principio del interés superior del niño. ${ }^{22}$

update/Spanish_DSM5Update2016.pdf, visitada: 31 de julio de 2019. En el DSM- 1 y 2 el "autismo" era considerado un síntoma más de la esquizofrenia. El DSM-3, publicado en 1980, cambió el enfoque diagnóstico de los trastornos mentales. Hasta entonces, los TEA eran considerados estados psicológicos y, a partir de este momento, pasaron a definirse como "categorías de enfermedad" y se incorpora por primera vez el término "autismo infantil". Luego, en el año 1987, existió una versión revisada del DSM del año 1980 que empleó la categoría de "trastornos generalizados del desarrollo", aunque suprimió el término "autismo infantil" de este grupo, reemplazándolo por el de "trastorno autista", puesto que, los síntomas continuaban aún en la etapa adulta. Posteriormente el DSM-4 (1994) y el DSM 4-TR (2000) definieron el "trastorno autista" bajo tres categorías diagnósticas: alteración en la interacción social; variación en la comunicación; y presencia de patrones de comportamiento, intereses y actividades restringidas, repetitivas y estereotipadas. Con el DSM-5 (2013) todos los subtipos del llamado "autismo" quedaron circunscritos en una sola categoría: "TEA", la que reemplazó el término "trastornos generalizados del desarrollo". Véase: Bonilla y ChASKel, cit. (n. 19), p. 2.

${ }^{21}$ Corte de Apelaciones de Antofagasta, 6 de diciembre de 2018, Rol No3.149-2018.

${ }^{22} \mathrm{Se}$ indicaron en la acción de protección otros fundamentos que nos limitaremos a mencionar, dado que en este comentario nos centraremos en el análisis del acto del recurrido, en la medida que puede ser calificado de arbitrario y/o ilegal a la luz del principio del interés superior del niño. En efecto, en concepto del recurrente, la comunicación emitida por la entidad educacional carecía de motivación, 
El Colegio Chuquicamata al evacuar su informe contravino los hechos narrados. En dicho sentido, según el considerando $2^{\circ}$ del fallo en comento, indicó que existía un error, dado que de ninguna manera "la decisión se ha sustentado en alguna medida disciplinaria producto de alguna transgresión al Manual o Reglamento de Convivencia, por el contrario, se sustenta en la situación de aprendizaje del menor en la educación parvularia, que no ha presentado avances desde su ingreso, velando por no atentar contra su derecho a acceder a una educación especializada de acuerdo al diagnóstico que presenta". Es decir, la expulsión contribuiría a una mejor educación para el niño objeto de dicha medida, por lo que tal acto beneficiaría a su propio interés.

El recurrido indicó, además, según se aprecia en el considerando $3^{\circ}$, que se sostuvieron reuniones con los apoderados del niño, con la educadora diferencial y con una fonoaudióloga y que fruto de lo anterior se entregó la evaluación de la adaptación curricular del niño expulsado. En virtud de lo anterior, concluyó el recurrido que S.E.N.A. solo había "logrado avances rutinarios y repetitivos, y que requiere trabajar con un currículo específico a su diagnóstico, situación que el colegio en su calidad de científicohumanista no puede ofrecer, sumado a que este año el apoderado presentó certificado médico en que se diagnostica al estudiante con trastornos del sueño, de integración sensorial, sugiriendo la neuróloga tratante educación diferenciada, musicoterapia, terapia fonoaudiología permanente $\mathrm{y}$ ocupacional".

pues no se mencionaba ninguna de las causales de no renovación o cancelación de matrícula descritas en el reglamento de convivencia escolar, vulnerándose, de este modo, el debido proceso, pues no se dio cumplimiento a las causales establecidas en la propia reglamentación que vinculaba al recurrido. Según el artículo 2 de la Ley $\mathrm{N}^{\circ}$ 19.979, publicada en el D.O. de fecha 6 de noviembre de 2004, se modificó el DFL N ${ }^{\circ} 2$ del Ministerio de Educación, publicado en el D.O. el 28 de noviembre de 1998, agregándose al artículo 6 de dicha normativa una letra d), en cuya virtud los establecimientos deben contar con un reglamento interno que rija las relaciones entre el establecimiento, los alumnos, los padres y apoderados. En dicho reglamento se deberán señalar: las normas de convivencia en el establecimiento; las sanciones y reconocimientos que origina su infracción o destacado cumplimiento; los procedimientos por los cuales se determinarán las conductas que las ameritan; y, las instancias de revisión correspondientes. Siempre en concepto del recurrente, se violaría, también, el derecho de propiedad sobre la matrícula educacional que tendría como titular a S.E.N.A, derecho que se encontraría incorporado a su patrimonio. En apoyo de lo anterior, se señaló que durante los tres años de permanencia de S.E.N.A en el Colegio Chuquicamata nunca fue objeto de sanción alguna. 


\section{Decisión del sentenciador}

La Corte de Apelaciones de Antofagasta resolvió acoger la acción de protección presentado en contra del Colegio Chuquicamata. La fundamentación de lo resuelto por el tribunal de segunda instancia estuvo centrada tanto en la arbitrariedad como en la ilegalidad del acto de expulsión.

A propósito de la arbitrariedad, indicó la Corte de Antofagasta, que la oportunidad para presentar las argumentaciones para negar el ingreso de S.E.N.A. al establecimiento educacional estaba constituida por la correspondiente fase de admisión, en consecuencia, con posterioridad a esta etapa el rechazo deviene en arbitrario. A mayor detalle, en el considerando $6^{\circ}$ se expresó: "el recurrido conocía desde el ingreso del niño a su establecimiento educacional que éste presentaba espectro de autismo, lo que implicaba e implica necesidades que requerían de una atención especial y/o distinta respecto de sus otros educandos, pese a lo cual lo aceptó e incorporó a su proyecto educativo en el año 2016 según la recurrente, desde 2015 según el recurrido, sea uno u otro, hace por lo menos, tres años lectivos, de modo tal que habiéndolo incorporado a su proyecto educacional con conocimiento de su particular situación, todas las argumentaciones para no renovar o para cancelar su matrícula en la actualidad devienen en arbitrarias o caprichosas porque cualquier negativa de matrícula o ingreso al establecimiento educacional debió efectuarse cuando se requirió el ingreso, puesto que era ésta y no otra, la oportunidad para hacer presente las supuestas falencias de un sistema de integración escolar". Agregó el sentenciador que: "Pese a ello el recurrido admitió al niño y aún más, reconoce que adaptó el sistema curricular a las necesidades del niño durante los tres años de permanencia, lo que demuestra que aceptó asumir la educación del niño conociendo su situación especial". En efecto, el recurrido implementó un Plan de Adecuación Curricular Individual que benefició al niño. Siempre en el considerando $6^{\circ}$, dejó constancia la Corte que S.E.N.A. sí obtuvo algunos avances en su aprendizaje. Por lo dicho, se estableció en el considerando $8^{\circ}$ que era discriminador el actuar del recurrido.

Luego, razonó el sentenciador sobre la ilegalidad del acto de expulsión, basándose para ello en los artículos 34 y 36 de Ley No 20.422. En conformidad al tenor del primer artículo, "Los establecimientos de educación parvularia, básica y media contemplarán planes para alumnos con necesidades educativas especiales y fomentarán en ellos la participación de todo el plantel de profesores y asistentes de educación y demás integrantes 
de la comunidad educacional en dichos planes". En la misma dirección, el artículo 36 dispone: "Los establecimientos de enseñanza regular deberán incorporar las innovaciones y adecuaciones curriculares, de infraestructura y los materiales de apoyo necesarios para permitir y facilitar a las personas con discapacidad el acceso a los cursos o niveles existentes, brindándoles los recursos adicionales que requieren para asegurar su permanencia y progreso en el sistema educacional. Cuando la integración en los cursos de enseñanza regular no sea posible, atendida la naturaleza y tipo de la discapacidad del alumno, la enseñanza deberá impartirse en clases especiales dentro del mismo establecimiento educacional o en escuelas especiales".

Es decir, los establecimientos educacionales no pueden excusarse de continuar brindando sus servicios a NNA que tengan necesidades particulares, aun cuando no dispongan de programas de intervención especializada, pues la ley mandata que se contemplen planes para estudiantes con las mencionadas necesidades educativas.

\section{Arbitrariedad e ilegalidad del acto realizado por el recurrido}

Concordamos con lo resuelto por la Corte de Apelaciones de Antofagasta. En efecto, la medida de expulsión adoptada por el recurrido permite fundamentar y aceptar desde una doble perspectiva la acción de protección que genera este comentario. Es decir, apreciamos tanto una arbitrariedad como una ilegalidad en la conducta del recurrido, todo lo que trae como consecuencia una afección al principio del interés superior del niño. Adelantamos que, muy interesante y sagaz nos parece la defensa del Colegio Chuquicamata relativa a que la expulsión, en la especie, permitiría cautelar adecuadamente el nombrado interés.

\section{a) De la arbitrariedad de la medida de expulsión}

La noción de "arbitrario" del diccionario de la RAE define el referido concepto del modo siguiente: "sujeto a la libre voluntad o al capricho antes que a la ley o a la razón". ${ }^{23}$ En otros términos, como es sabido, una conducta podrá ser calificada de "arbitraria" en la medida que no posea un fundamento centrado en la razón. Pensamos que lo señalado puede predicarse respecto

\footnotetext{
${ }^{23}$ Real Academia Española, Diccionario de la lengua española, $23^{\mathrm{a}}$ Ed., Versión 23.3 en línea, https:// dle.rae.es, consultada: 29 de julio de 2019.
} 
del hecho que ha motivado la litis que analizamos. En efecto, el recurrido ha manifestado como justificación de su accionar consideraciones vinculadas al interés superior del niño. Así, la medida de expulsión implicaría evitar la causación de una vulneración del interés superior, pues facilitaría el ejercicio del derecho de S.E.N.A. de acceder a una educación especializada en atención a su diagnóstico.

No estamos de acuerdo con los planteamientos del recurrido, por el contrario, pensamos que la medida de expulsión contradice el supuesto interés por el bienestar del NNA que alegó buscar el Colegio Chuquicamata. Avanzando en esta línea de razonamiento, una auténtica protección del principio del interés superior del niño exige cautelar "the best interest of the child", siguiendo la expresión empleada por la versión en inglés de la Convención sobre los Derechos del Niño. Así, pareciera de toda evidencia que el mejor interés del niño recomienda no expulsarle del recinto en el que ha realizado sus estudios, salvo que concurran causas especialmente calificadas. Pero nuestro análisis quedaría trunco si no se incluye la variante que caracteriza la sentencia que comentamos, es decir, la influencia del TEA padecido por S.E.N.A., en consecuencia, es particularmente grave el daño provocado al principio del interés superior al expulsar a un niño que presenta las particularidades propias del aludido trastorno, las que se manifiestan en una serie de deficiencias en los aspectos del neurodesarrollo, a las que ya nos hemos referido.

Súmese a lo anterior que, el interés superior del niño requiere evitar los cambios que modifiquen su entorno, tal como lo reconoce la Corte en el considerando $9^{\circ}$, ya sea en términos emocionales o materiales, que se traduzcan en repercusiones negativas para el desarrollo y futuro del NNA. Esta interpretación del interés superior creemos es la que debe primar, pues para el especial caso del TEA las condiciones del ambiente resultan fundamentales, dado que, "el entorno, los estímulos sensoriales, cambios inesperados de rutina, estrés ambiental, falta de estructura, excesivas demandas que no pueden cumplir, negaciones, malinterpretación de su entorno y situaciones que provocan ansiedad y miedo, suelen precipitar los problemas de conducta". ${ }^{24}$ Lo último claramente ocurriría si se concretara el acto de expulsión, en cuyo evento, por lo tanto, se conculcaría el interés superior de S.E.N.A, el que implica en la especie recibir una educación

\footnotetext{
${ }^{24}$ Hervás, Amaia; Rueda, Isabel, "Alteraciones de conducta en los trastornos del espectro autista", Revista de Neurología, 2018, Vol. 66, №. 1, p. 534.
} 
en un colegio tradicional que cuente con el personal idóneo para apoyar en la adaptación de los planes curriculares y en el proceso de evaluación atendiendo sus especiales necesidades educativas.

En nuestra opinión, lo realmente buscado por el recurrido no era potenciar el futuro desarrollo del niño, sino que, sobre la base de su pura voluntad, decidió no tomar las medidas necesarias para tratar de un modo adecuado los requerimientos de un niño que presenta un TEA y por sobre ello, prefirió no renovarle la matrícula, esto es, expulsarle.

Pensemos en lo siguiente: ¿qué exige la razón en un caso como el estudiado? Según nuestro entender, una conducta basada en la razón implica que el propio recurrido hubiese cambiado y/o mejorado sus procedimientos de forma tal de poder satisfacer el mejor interés de los alumnos que presenten características particulares. La razón, además, lleva a rechazar un acto que traslada al propio niño y a sus padres los perjuicios derivados de soportar un cambio de entidad como lo es el adaptarse a un nuevo recinto educacional, con todo lo que ello implica.

Finalizamos esta parte indicando que concordamos con lo señalado por la Corte de Apelaciones de Antofagasta respecto al momento de la expulsión. En efecto, en el caso que comentamos el factor cronológico cobra particular relevancia: S.E.N.A fue admitido voluntariamente por el recurrido, a sabiendas de su particular condición, por lo que no resulta procedente que, con posterioridad, basándose en las mismas condiciones, se tome una medida contradictoria con el propio actuar inicial del recurrido. Además, es importante tener presente que S.E.N.A. nunca fue sancionado por el recurrido, cuestión que contribuye a calificar su accionar como alejado de un fundamento de razón.

En suma, la razón dirige, primero, a afirmar que un determinado colegio no puede expulsar a un niño admitido en su momento conociendo que éste presentaba un TEA basándose en la propia existencia de dicho trastorno $\mathrm{y}$, en segundo lugar, también conduce a transitar por un sendero opuesto al elegido por el recurrido, o sea, el propio recinto de educación debe adaptarse a las necesidades especiales de sus alumnos.

\section{b) Ilegalidad del acto del recurrido}

El acto del recurrido, además de arbitrario, es ilegal. Antes de profundizar dicha afirmación, es pertinente tener presente los objetivos de los establecimientos de educación, en particular, con respecto a los NNA con necesidades especiales. 
En virtud del artículo 10 letra a) de la Ley $20.370,{ }^{25}$ Ley General de Educación, los establecimientos educacionales deben otorgar una atención adecuada y oportuna, en el caso de NNA con necesidades especiales, además, los estudiantes no deben ser discriminados arbitrariamente. Agrega el inciso $1^{\circ}$ del artículo 23 de la nombrada Ley, que los establecimientos regulares deben proveer un conjunto de servicios, recursos humanos, técnicos, conocimientos especializados y ayudas para atender las necesidades educativas especiales de sus alumnos a lo largo de su escolaridad.

Por otro lado, la Ley $\mathrm{N}^{\circ} 20.422,{ }^{26}$ que establece normas sobre igualdad de oportunidades e inclusión social de personas con discapacidad, en el artículo 35, indica que la educación especial es una modalidad del sistema escolar que provee servicios y recursos especializados, para asegurar aprendizajes de calidad a NNA con necesidades educativas especiales asociadas o no a una discapacidad, asegurando el cumplimiento del principio de igualdad de oportunidades para todos los educandos.

Es menester analizar lo dispuesto en los artículos 34 y 36 de la Ley $\mathrm{N}^{\mathrm{o}}$ 20.422. Los citados preceptos establecen para los establecimientos de educación deberes relativos al modo de proceder respecto de los alumnos con condiciones especiales, no simples facultades, de ahí que el contenido de tales normas sea vinculante para las mencionadas personas jurídicas. En total, tres son los deberes regulados en las normas que ahora analizamos, dos de ellos se encuentran en el artículo 34 y otro en el artículo 36.

En el primer precepto se consagran los siguientes deberes: primero, elaborar planes para alumnos con necesidades educativas especiales y, en segundo lugar, fomentar la participación en dichos planes de todo el plantel de profesores, asistentes de educación y, en general, del resto de los integrantes de la comunidad educacional. Como podemos advertir, estos dos primeros deberes se centran en la faceta de debida y adecuada preparación del establecimiento educacional con miras a recibir y permitir la continuidad del proceso de aprendizaje de alumnos que tengan características peculiares. Así, en el primer deber, lo anterior se concreta en la elaboración de planes especializados, mientras que el segundo, en la participación en la creación de tales planes por parte de la comunidad educacional en general. El sentenciador no realizó mención alguna a la violación de estos deberes, cosa diversa aconteció con el restante deber.

\footnotetext{
${ }^{25}$ Ley N $\mathrm{N}^{\circ} 20.370,2009$.

${ }^{26}$ Ley No 20.422, 2010.
} 
En el artículo 36, como tercer deber, se indica que corresponde a los recintos educacionales incorporar las innovaciones y adecuaciones que fuesen necesarias en materia curricular, de infraestructura y proporcionar los materiales de apoyo adecuados para "permitir" y "facilitar" a las personas con necesidades especiales el acceso a los cursos o niveles existentes, disponiendo para lo anterior de los recursos adicionales que se requieran para asegurar su permanencia y progreso en el sistema educacional. Sin perjuicio de lo señalado, si la integración en los cursos de enseñanza regular no fuese posible, en virtud de la naturaleza y tipo de la discapacidad del alumno, la enseñanza deberá impartirse en clases especiales dentro del mismo establecimiento educacional o en otras escuelas idóneas para ello.

El tercer deber es particularmente importante para efectos de determinar la ilegalidad del acto de expulsión. Lo dicho, dado que este deber no se limita solamente a prescribir la obligatoriedad de aplicar, en una serie de ámbitos, las medidas de inclusión que fuesen pertinentes para hacer factible el acceso a los cursos que componen el respectivo programa de estudios, sino que, además de "permitir", emplea el verbo "facilitar". Esto es, consagra una doble imposición: hacer "viable" y "sencillo" el cumplimiento de lo anterior. Este deber resultó conculcado por el recurrido, dado que, en una primera etapa, por tres años, reconoció que adaptó el sistema curricular a las necesidades de S.E.N.A., pero también admitió que con posterioridad dejó de implementar medidas tendientes a permitir la continuación de su matrícula. En efecto, no es posible apreciar esfuerzo alguno en dicho sentido, toda vez que optó voluntariamente por no renovar la matrícula. ${ }^{27}$

Con todo, en ciertos supuestos, no obstante, las buenas intenciones de la comunidad de un determinado colegio, el legislador admite que puede resultar inviable que algunos NNA puedan integrarse al procedimiento ordinario de enseñanza, es decir, la realidad puede tornar en impracticable la observancia del tercer deber. En tales casos, es necesario acudir a clases especiales impartidas en el mismo recinto o en otro diverso mejor capacitado para ello. Es interesante advertir que el núcleo de la defensa del recurrido (el interés superior del niño compele a que continúe sus estudios en un colegio especializado en tratar a NNA con características particulares) pudo

\footnotetext{
${ }^{27}$ Se indicó en el considerando $6^{\circ}$ de la sentencia comentada: "El establecimiento se encuentra legalmente obligado a mantener un programa de integración escolar para niños con discapacidades y necesidades especiales, para lo cual debe hacer lo que precisamente efectuó durante los tres años en los que S.E.N.A ha estado inserto en su establecimiento educacional".
} 
haber sido mejor planteado, desarrollando y probando este punto. Pero, si bien es cierto que se hizo presente lo anterior, no se rindió probanza alguna tendiente a acreditar la imposibilidad por parte del recurrido de otorgar la educación que necesitaba el niño.

Lo último ha sido aplicado por la Corte de Apelaciones de Santiago, tribunal que conociendo una acción de protección deducida por una apoderada en representación de su hija expulsada que padecía de un trastorno de lenguaje, falló lo que sigue: "que así las cosas la decisión de la recurrida aparece racional y apoyada en motivaciones suficientes que resultan idóneas y fundada en la normativa legal señalada, que en todo caso resulta ser protectora de los derechos de la niña E., que requiere de apoyo y ayuda adicional en su proceso educativo; dado que se encuentra acreditado que ella presenta necesidades educativas especiales; todo ello sin perjuicio que la presente acción ha perdido oportunidad toda vez que la menor ya está asistiendo a un establecimiento educacional; por todo lo cual el recurso no podrá prosperar". ${ }^{28}$

Otra sentencia que recorre la senda comentada fue la dictada por la Corte de Apelaciones de Valdivia. En este juicio, razonó el tribunal de alzada que, ante los nulos avances obtenidos por un niño que tenía un trastorno generalizado del desarrollo, la decisión del establecimiento de cancelar la matrícula era ajustada a derecho. En tal sentido, se resolvió que: "se ha podido observar que se han brindado las herramientas pertinentes para cubrir las necesidades especiales del menor, sin que ello haya significado una evolución en su proceso de desarrollo educativo y social, como se refleja de las constancias existentes en este proceso de las entrevistas efectuadas y los informes elaborados por los profesionales del establecimiento recurrido, rolantes a fojas 7 a 16 y de fojas 180 a 206, los que apreciados según las normas de la sana crítica, no logran establecer que la recurrida haya actuado en desprecio del derecho a la igualdad, consagrado en el artículo $19 \mathrm{~N}^{\circ} 2$ de la Constitución Política de la República o cometido actos discriminatorios". ${ }^{29}$

En suma, teniendo en vista lo señalado, incluso en el evento de un eventual desempeño educativo deficiente por parte de S.E.N.A presuntamente atribuible al trastorno que le afectaba, en estricta sujeción al tercer deber del artículo 36 de la Ley $\mathrm{N}^{\mathrm{o}}$ 20.422, la medida de expulsión emanada del recurrido necesariamente debe ser calificada de ilegal.

\footnotetext{
${ }^{28}$ Corte de Apelaciones de Santiago, 17 de abril de 2015, Rol No82.836-2014, considerando $8^{\circ}$.

${ }^{29}$ Corte de Apelaciones de Valdivia, 12 de marzo de 2015, Rol №86-2015, considerando $15^{\circ}$.
} 


\section{Arbitrariedad del recurrido: ¿y la responsabilidad de los padres?}

La sentencia que comentamos fue confirmada con declaración por la Corte Suprema, ${ }^{30}$ que reprodujo la resolución apelada, salvo los considerandos $6^{\circ}, 7^{\circ}, 8,9^{\circ}$ y $10^{\circ}$, los que fueron eliminados. Así, una parte fundamental de los argumentos empleados por la Corte de Apelaciones de Antofagasta fueron desestimados sin indicarse razones para ello. ${ }^{31}$ Con todo, los argumentos de ambas sentencias son valiosos, dado que contribuyen a dotar de contenido al principio del interés superior del niño, como veremos a continuación. Lo señalado debe resaltarse teniendo en vista las características especiales del caso que comentamos.

Para la Corte Suprema fue decisivo, según se estableció en el considerando $3^{\circ}$, que en el Proyecto Educativo Institucional del recurrido se consigna como valor la tolerancia, lo que implica empatía, reconocer al otro como un legítimo diferente, con diversa cultura, religión, manera de pensar y de actuar. Súmese a lo anterior, como se indicó en el considerando $4^{\circ}$, que el recurrido implementó en favor de S.E.N.A. un Plan de Adecuación Curricular Individual, el que consideró el TEA padecido por éste. Lo último concuerda con el suprimido considerando $6^{\circ}$ de la sentencia de la Corte de Apelaciones de Antofagasta. Por lo señalado, entendió la Corte Suprema que el recurrido sí contaba con algunos medios para tratar las particulares necesidades de S.E.N.A.

También fue decisivo lo expresado en el considerando $5^{\circ}$ por la consejera técnico del Centro de Medidas Cautelares de Santiago, quien se manifestó contraria a la expulsión de S.E.N.A. A mayor detalle, dicha consejera señaló: "en el caso de los niños con trastorno del espectro autista moderado las habilidades pre académicas se desarrollan lentamente, evidenciándose notablemente reducidas en comparación de sus iguales, es

\footnotetext{
${ }^{30}$ Corte Suprema, 6 de junio de 2019, Rol No 32.952-2018.

${ }^{31}$ Recordemos que el considerando $6^{\circ}$ construía la arbitrariedad sobre la base de que el recurrido, a la época de admitir a SENA conocía su TEA, además, en dicho considerando se estableció que el recurrido sí poseía un programa de inserción escolar y que se observaron avances en el proceso de aprendizaje. Por su parte, el considerando $7^{\circ}$ entendió vulnerados los artículos 34 y 36 de la Ley N ${ }^{\circ}$ 20.422 , en cuya virtud los establecimientos educacionales deben implementar y mantener programas especiales para NNA con discapacidades y necesidades especiales. El considerando $8^{\circ}$ insistía en el trato discriminatorio otorgado a SENA. En el considerando $9^{\circ}$ se señaló que el acto del recurrido afectaba el interés superior del niño, al separarlo de los afectos, amistad y relaciones de convivencia existentes en el recinto de educación en el que estudiaba. Finalmente, en el considerando $10^{\circ}$, se indicó que por todo lo anterior, sólo cabía acoger el recurso.
} 
así como el lenguaje es menos complejo que el de otros niños de su edad. Sin embargo, el desempeño en la capacidad de relación se vincula con su formación familiar y su entorno". Agregó la consejera que, "en consideración a su diagnóstico es fundamental priorizar su escolarización en lugar de privarlo de ella, toda vez que esto último resulta más arriesgado para el desarrollo del niño que permanecer en un colegio que no cuente con todas las condiciones para sus necesidades y que la exclusión del colegio al que acude actualmente, que es su entorno permanente, mermará su autoestima y seguridad".

Por todo lo anterior, tal como se consignó en el considerando $6^{\circ}$, se determinó la arbitrariedad del actuar del recurrido, el que careció de fundamentos y contradijo los valores institucionales de su propio Proyecto Educativo. Centrándose en el principio del interés superior del niño, se añadió en el considerando mencionado que, la no renovación de matrícula causaría daño a S.E.N.A., al alejarlo del espacio físico y humano que le era conocido, lo que afectaría su desarrollo, autoestima y seguridad, especialmente considerando su situación de salud. Como podemos advertir, en esta parte la Corte Suprema coincide con lo resuelto en el considerando $9^{\circ}$ de la sentencia de la Corte de Apelaciones de Antofagasta, aunque, como ya indicamos, dicho considerando fue suprimido por nuestro supremo sentenciador.

Relevante es el considerando $7^{\circ}$ de la sentencia de la Corte Suprema, dado que establece que en el proceso educativo formal debe existir una constante comunicación y colaboración mutua entre los padres y el recinto educacional. Por lo dicho, se señala que:

"En consecuencia, los padres del niño S.E.N.A. son los principales encargados de que éste tenga acceso a los tratamientos profesionales neurológicos, fonoaudiológico, psiquiátrico, psicológicos, de terapia educacional y kinesiológico, que son fundamentales en su desarrollo y que complementan la labor del colegio".

Los argumentos indicados permitieron a la Corte Suprema establecer que el actuar del recurrido fue arbitrario y contrario a la igualdad ante la ley, por lo que en el considerando $8^{\circ}$ se confirmó la resolución apelada con "declaración que se dispone, además de la renovación de matrícula para el año 2019 del niño S.E.N.A., que los padres de éste deberán encargarse que acceda a la brevedad a los tratamientos profesionales neurológicos, fonoaudiológico, psiquiátrico, psicológicos, de terapia educacional y kinesiológico necesarios para tratar su diagnóstico y debidamente 
determinados por los especialistas respectivo".

Como podemos apreciar, la Corte Suprema resolvió que, además de la labor de los establecimientos educacionales, es fundamental que los padres adopten las medidas pertinentes para intentar obtener un adecuado tratamiento de las particulares necesidades educacionales de sus hijos. En pocas palabras, el recurrido no es el único que debe preocuparse de lo anterior. Los considerandos $7^{\circ}$ y $8^{\circ}$ concuerdan con lo establecido en el artículo 222 del Código Civil, inciso $1^{\circ}$, en cuya virtud, "La preocupación fundamental de los padres es el interés superior del hijo, para lo cual procurarán su mayor realización espiritual y material posible (...)".

Mismo sendero ha recorrido la doctrina al referirse expresamente al rol de la familia de los NNA que padecen un TEA, indicándose que a ésta le compete una función de la mayor relevancia "al ser el grupo primario más representativo y de referencia social. Específicamente en el caso del TEA pasa a ser imprescindible su aportación educativa, puesto que facilita los apoyos y oportunidades para el desarrollo de las personas más allá de sus alteraciones y dificultades". ${ }^{32}$ Lo anterior sigue lo establecido en la Convención de los Derechos del Niño, pues desde su "preámbulo, hace referencia a la familia como núcleo fundamental de formación y desarrollo personal, emocional y social en un ambiente de amor y acogimiento en tanto los niños, las niñas y los adolescentes maduran y adquieren la mayoría de edad". ${ }^{33}$

Hemos visto que tanto la Corte de Apelaciones de Antofagasta como la Corte Suprema calificaron de arbitraria la conducta del recurrido, pero no por los mismos fundamentos, aunque coincidieron en el daño derivado de dicho actuar para el interés superior de S.E.N.A. Con todo, la principal diferencia radica en que el primer sentenciador, además, entendió que se había producido una ilegalidad al infringirse los artículos 34 y 36 de la Ley $N^{0}$ 20.422. Por su parte, la Corte Suprema se centró en la actuación arbitraria del recurrido y en el deber de los padres de procurar una adecuada satisfacción de las necesidades de salud de sus hijos, sin mencionar los referidos artículos.

En nuestra opinión, acertado fue el razonamiento de la Corte de

\footnotetext{
${ }^{32}$ BAÑa, cit. (n. 12), p. 323.

${ }^{33}$ Pérez Contreras, María de Montserrat, "El entorno familiar y los derechos de las niñas, los niños y los adolescentes: una aproximación”, Boletín Mexicano de Derecho Comparado, 2013, Vol. 46, № 138, p. 1153.
} 
Apelaciones de Antofagasta al estimar, además, que se había cometido una ilegalidad, dado que, como hemos expuesto, es deber de los recintos educacionales gestionar y/o adecuar los recursos pertinentes con el objetivo de permitir la educación de NNA con necesidades especiales. Así, en la especie, la afección al interés superior de S.E.N.A. pudo haber sido mejor fundada sobre la base tanto de la arbitrariedad como de la ilegalidad del acto impugnado. Lo dicho no supone desconocer el rol de primer orden que corresponde a los padres en el cuidado de la salud de sus hijos.

En definitiva, destacamos la relevancia de las dos sentencias al aportar a la construcción del interés superior en el caso comentado. En este sentido: ambas resoluciones entendieron que es arbitrario no renovar la matrícula de un NNA que tiene un TEA (y que ello provoca una serie de efectos negativos); la Corte de Antofagasta agregó la consideración de la violación de determinados deberes de fuente legal; y la Corte Suprema añadió el rol protagonista que deben representar los padres en la búsqueda del bienestar de sus hijos y/o hijas.

\section{BIBLIOGRAFÍA CITADA}

\section{a) Doctrina}

Aguilar, Gonzalo, "El principio del interés superior del niño y la Corte Interamericana de Derechos Humanos", Estudios Constitucionales, 2008, Vol. 6, $\mathrm{N}^{\circ} 1$.

Artigas, Joseph y Paula, Isabel, "El autismo 70 años después de Leo Kanner y Hans Asperger”, Revista Especializada Neuropsiquiatría, 2011, $\mathrm{N}^{\mathrm{o}}$ 115, Vol. 32.

Asociación Americana de Psiquiatría (Eds.), Guía de consulta de los criterios diagnósticos del DSM 5, American Psychiatric Publishing, Arlington, 2014, enm línea: http://www.eafit.edu.co/ninos/ reddelaspreguntas/Documents/dsm-v-guia-consulta-manual-diagnosticoestadistico-trastornos-mentales.pdf, consultada: 27 de julio de 2019.

Asociación Americana de Psiquiatría (Eds.), Diagnostics and Statistics Manual of Mental Disorders, American Psychiatric Publishing, Arlington, 2016, en línea: https://dsm.psychiatryonline.org/pb-assets/dsm/ update/Spanish_DSM5Update2016.pdf, visitada: 31 de julio de 2019. 
BAÑA, Manoel, "El rol de la familia en la calidad de vida y la autodeterminación de las personas con trastorno del espectro del autismo", Ciencia Psicológica, 2015, Vol. 9, № 2.

BARCIA, Rodrigo, "La evolución de la custodia unilateral conforme a los principios de interés superior del niño y corresponsabilidad de los padres", Ius et Praxis, 2018, No 2 .

Bonilla, María; Chaskel, Roberto, "Trastorno del Espectro Autista", Curso Continuo de Actualización de Pediatría: Sociedad Colombiana de Pediatría, 2016, Vol. 15, № 1 .

Cillero, Miguel, "El interés superior del niño en el marco de la Convención internacional sobre los Derechos del Niño", Justicia y Derechos del Niño, 1999, № 1.

GonzÁlez, Yanesy, "La interdisciplinariedad en la investigación como principio de la responsabilidad social universitaria", Revista Congreso Universitario, 2017, Vol. 6, $\mathrm{N}^{\mathrm{o}} 4$.

Hervás, Amaia; Rueda, Isabel, "Alteraciones de conducta en los trastornos del espectro autista", Revista de Neurología, 2018, Vol. 66, № 1 .

Lathrop, Fabiola, Cuidado personal de los hijos. Análisis de la Ley de matrimonio civil y Tribunales de Familia, Punto Lex, Santiago, 2005.

Lepin, Cristian, "Los nuevos principios del Derecho de Familia", Revista Chilena de Derecho Privado, 2014, № 23, pp. 9-55.

Martínez, Agustín; Piqueras, José, "Conductas repetitivas en personas con trastorno del espectro autista: Relación con los antecedentes familiares y el tratamiento farmacológico", Revista Nacional e Internacional de Educación Inclusiva, 2017, $\mathrm{N}^{\circ}$ 1, Vol. 10.

Ministerio de Educación de Chile (Eds.), Guía de apoyo técnico pedagógico para necesidades educativas especiales en el nivel de educación parvularia, Unidad de Educación Especial, Santiago, 2008, 1era Ed., en línea: http://especial.mineduc.cl/wp-content/uploads/sites/31/2016/08/ GuiaAutismo.pdf, consultada: 03 de julio de 2019.

Pérez Contreras, María de Montserrat, "El entorno familiar y los derechos de las niñas, los niños y los adolescentes: una aproximación", Boletín Mexicano de Derecho Comparado, 2013, Vol. 46, № 138.

Ravetllat, Isaac; Pinochet, Ruperto, "El interés superior del niño en el marco de la Convención Internacional sobre los Derechos del Niño y su configuración en el Derecho civil chileno", Revista Chilena de Derecho, 2015, Vol. 42, No 3.

Real Academia Española, Diccionario de la lengua española, $23^{\mathrm{a}}$ Ed., 
Versión 23.3, en línea: https://dle.rae.es, consultada: 29 de julio de 2019.

SilveirA, Israel, "La aplicación del interés superior del niño en el ámbito educativo. Las concepciones de los profesionales de los equipos de orientación escolar". XXIII Jornadas de Investigación. XII Encuentro de Investigadores en Psicología del MERCOSUR. Facultad de Psicología Universidad de Buenos Aires, Buenos Aires, 2016, en línea: https://www. aacademica.org/000-044/455.pdf.

b) Jurisprudencia

Corte de Apelaciones de Antofagasta, 6 de diciembre de 2018, Rol N ${ }^{\circ}$ 3.149-2018.

Corte de Apelaciones de Valdivia, 12 de marzo de 2015, Rol № 862015.

Corte de Apelaciones de Santiago, 17 de abril de 2015, Rol N 82.8362014.

Corte Suprema, 6 de junio de 2019, Rol No 32.952-2018.

Corte Suprema, 18 de mayo de 2016, considerando $11^{\circ}$, Rol $\mathrm{N}^{\circ}$ 35.522-2015.

Corte Suprema, 27 de mayo de 2015, considerando $5^{\circ}$, Rol N $29.566-$ 2014

c) Normativa

Convención Internacional de los Derechos del Niño, ONU, 1989.

Comité de Derechos Económicos, Sociales y Culturales ONU, Observación $N^{\circ} 11$ (1999), Planes de acción para la enseñanza primaria (artículo 14 del Pacto), Naciones Unidas, Nueva York, 2000.

Comité de Derechos del Niño UNICEF, Observación General $N^{\circ} 1$ (2001), Propósitos de la educación, Naciones Unidas, Nueva York, 2001.

Comité de Derechos del Niño UNICEF, Observación General $N^{\circ}$ 14 (2013), sobre el derecho del niño a que su interés superior sea una consideración primordial (artículo 3, párrafo 1), Naciones Unidas, Nueva York, 2013.

Ley $\mathrm{N}^{\circ} 20.370,12$ de septiembre de 2009.

Ley $\mathrm{N}^{\circ} 20.422,10$ de febrero de 2010. 